\title{
Inflammatory bowel disease: study of cell mediated cytotoxicity for isolated human colonic epithelial cells
}

\author{
B J KEMLER AND E ALPERT* \\ From the Medical Services (Gastrointestinal Unit), Massachusetts General Hospital and the Department \\ of Medicine, Harvard Medical School, Boston, Massachusetts, USA
}

SUMMARY A better understanding of the mechanism(s) of cell mediated toxicity for colon cells in vitro may help clarify the pathogenesis of inflammatory bowel disease (IBD). We have examined both the cytotoxicity of IBD peripheral blood mononuclear cells and the kinetics of induction of such toxicity by soluble plasma factors. Peripheral blood mononuclear cells from IBD patients were found to be cytotoxic for the colon cells. With the use of Chang cells, this cytotoxicity was shown not to be due to an increase in spontaneous cell mediated cytotoxicity. Colon cell toxicity in vitro did not correlate with site of disease or severity, but decreased toxicity appeared to be associated with in vivo steroid administration. Plasma from some IBD patients was capable of inducing normal peripheral blood mononuclear cells to be toxic to colon cells. This ability was not affected by steroid therapy. The induction capacity of IBD plasma was not associated with the presence of circulating immune complexes, as measured by Raji RIA, suggesting that large complement fixing complexes are not the inducing and directing factors. Unlike findings in other systems, induction could be demonstrated after a one hour preincubation of mononuclear cells with IBD plasma. The kinetics of induction are consistent with the hypothesis that either cytophilic antibody or small circulating immune complexes arm K cells for specific colon cell lysis.

The many alterations in the reactivity of the cellular and humoral elements of patients with inflammatory bowel disease (IBD) initially led to the hypothesis that the immune system is involved in the pathogenesis of IBD. To date, the most compelling support for this hypothesis remains the demonstration of the in vitro cytotoxicity of IBD peripheral blood lymphocytes (PBL) for trypsinised colon cells. ${ }^{1-3}$

The precise mechanism of this in vitro cytotoxicity, however, remains poorly defined. Although initial studies suggested that lymphocytes from patients with IBD were intrinsically committed to colon cell lysis, ${ }^{1}$ more recent work suggests that there may be an interaction between uncommitted effector cells and soluble 'arming' factors. ${ }^{45}$

It has been suggested that the soluble arming factor(s) could be either (1) cytophilic antibodies $(\mathrm{CAb})$ or (2) circulating immune complexes (CIC) having an antibody specificity for colon cell antigens.

*Address for correspondence: Dr Elliot Alpert, Gastrointestinal Unit, Massachusetts General Hospital, Boston, Massachusetts 02114, USA.

Received for publication 25 October 1979
Evidence for this hypothesis stems from a number of observations. First, CAb and CIC have been shown to be capable of inducing specific cytotoxicity in other in vitro systems. ${ }^{7}$ Second, a previous study has presented some data to suggest that colon cell toxicity induction is produced by a high molecular weight substance, the activity of which is blocked by an anti-immunoglobulin. ${ }^{5}$ Third, the effector cells in the in vitro reaction apparently possess surface receptors for the Fc portion of immunoglobulins, ${ }^{8}$ so that either CAb or CIC could theoretically bind to the effector cell and direct its activity. Finally, there is evidence to suggest that CIC occur in patients with IBD, ${ }^{910}$ and that the composition of these complexes may be disease specific. ${ }^{11}$

The kinetics of induction as presently delineated, however, appear inconsistent with the hypothesis that cytophilic antibody or circulating immune complexes initiate and direct cell mediated cytotoxicity for colon cells in vitro. Both cytophilic antibody and CIC generally produce rapid induction of cytotoxicity, ${ }^{6}{ }^{7}$ because the major requirement is binding of the immunoglobulin to the effector cells via $\mathrm{Fc}$ receptors. In contrast, in the previously re- 
ported cytotoxicity assay, induction of toxicity for colon cells was shown to occur only after four days of preincubation of normal PBL with IBD sera. ${ }^{4}$

There are many factors which influence the demonstration of cytotoxicity, such as the nature of the target cells, effector: target cell ratio, and the duration of the assay. As these assay parameters will also affect the detection of the induction of cytotoxicity, the long induction period previously reported might be an artefact of the assay conditions. Therefore, in the present study we developed a modification of the colon cell cytotoxicity assay to examine the ability of JBD peripheral blood mononuclear cells (PBMC) to produce colon cell toxicity. We studied the kinetics of the induction of cytotoxicity by IBD plasma, to determine if the time course is compatible with an induction mechanism involving either cytophilic antibody or circulating immune complexes. Finally, we looked for an association between the presence of CIC and the ability of plasma to induce cytotoxicity.

\section{Methods}

ISOLATION OF PERIPHERAL BLOOD

MONONUCLEAR CELLS (PBMC)

PBMC were obtained from 16 healthy controls and 20 IBD patients. The diagnosis of IBD was confirmed by a typical clinical history, contrast $x$-ray examinations, and histology; at least two of the three being positive in each case. Thirteen patients had ulcerative colitis and seven patients had Crohn's disease. Three of the CD patients had involvement limited to the small bowel by radiography and sigmoidoscopy. Six of the IBD patients had severe, 10 moderate, and four mild disease as defined by the criteria of Truelove et al..$^{12}$ and deDombal et al. ${ }^{13}$ for ulcerative colitis and Crohn's disease, respectively. Eleven of the 20 IBD patients were receiving corticosteroid therapy at the time of the study.

PBMC were isolated using the method of Boyum. ${ }^{14}$ Viable lymphocytes comprised $90 \%$ of the mononuclear cell population. Unless specifically noted, cells were maintained in Dulbecco's minimal essential media (DMEM) supplemented with $10 \%$ heat inactivated foetal calf serum, $100 \mathrm{U} / \mathrm{ml}$ penicillin, and $100 \mu \mathrm{g} / \mathrm{ml}$ streptomycin (complete media).

PREPARATION OF COLON EPITHELIAL

TARGET CELLS

Surgical specimens from patients undergoing colonic resection for malignancy were the source of epithelial cells. Immediately after resection of the colon, a $4 \times 4 \mathrm{~cm}$ piece of adjacent but normal mucosa was excised and placed in complete medium maintained at $4^{\circ} \mathrm{C}$. This was rapidly transferred to the laboratory where it was incubated in $1 \mathrm{mM}$ dithiothreitol (DTT) for 30 minutes at $25^{\circ} \mathrm{C}$. The specimens were placed on a dissection tray with the epithelial surface exposed and the remaining mucus and blood removed by flushing the mucosal surface with DMEM and very gentle brushing with forceps. The epithelial surface was removed by gentle scraping with a scapel blade, continually moistened with the complete medium containing 1\% collagenase (CLS 11, Worthington). The colon epithelial cell clumps were incubated in the collagenase DMEM solution for approximately three hours at $25^{\circ} \mathrm{C}$, with gentle agitation every 15 minutes. The epithelial cells were then washed several times with complete medium. and the cell number and viability were determined using a Neubauer haemocytometer, phase contrast microscopy (Fig. 1) and 0.4\% trypan blue exclusion. Approximately $2-3 \times 10^{6}$ epithelial cells could be obtained from a single specimen with greater than 97\% viability. Cytological examination with modified Papanicolaou stain and thin section microscopy confirmed the cells to be epithelial in origin.

\section{ASSAY FOR COLON CELL TOXICITY}

Colon epithelial target cells $\left(1 \times 10^{5}\right.$ in $150 \mu \mathrm{l}$ of complete medium) were placed in $2.5 \mathrm{ml}$ plastic Eppendorf tubes. Effector PBMC $\left(1 \times 10^{6}\right.$ in $150 \mu 1$ complete medium) were then added to the targets. Overnight incubation (10-12 hours) was performed in a $10 \% \mathrm{CO}_{2}$ incubator at $37^{\circ} \mathrm{C}$. In each experiment, control tubes consisted of $1 \times 10^{5}$ targets in a total volume of $300 \mu \mathrm{l}$ of complete medium. Heat inactivated test plasma, at a $15 \%$ concentration, was substituted for the foetal calf serum to examine direct plasma toxicity. All test and control reactions were run in triplicate. One hundred colon epithelial cells from each tube-that is, a total of 300 cells per effector or control incubation-were examined blindly for viability by trypan blue exclusion. Spontaneous colon cell death was approximately $10 \%$. Specific cytotoxicity was defined as the mean cell death occurring in the presence of specific effector cells or test plasma minus the mean spontaneous cell death occurring in the reactions containing only targets and complete medium. There was little variation in cytotoxicity of PBMC from a given individual. A normal individual tested against six different colon cell targets showed a range in cytotoxicity of only $2 \%$.

SPONTANEOUS CELL MEDIATED CYTOTOXICITY (SCMC)

Chang liver cells (Microbiological Associates), maintained in continuous tissue culture, were harvested by treatment with $0.25 \%$ trypsin and gentle scraping with a rubber policeman. These cells 


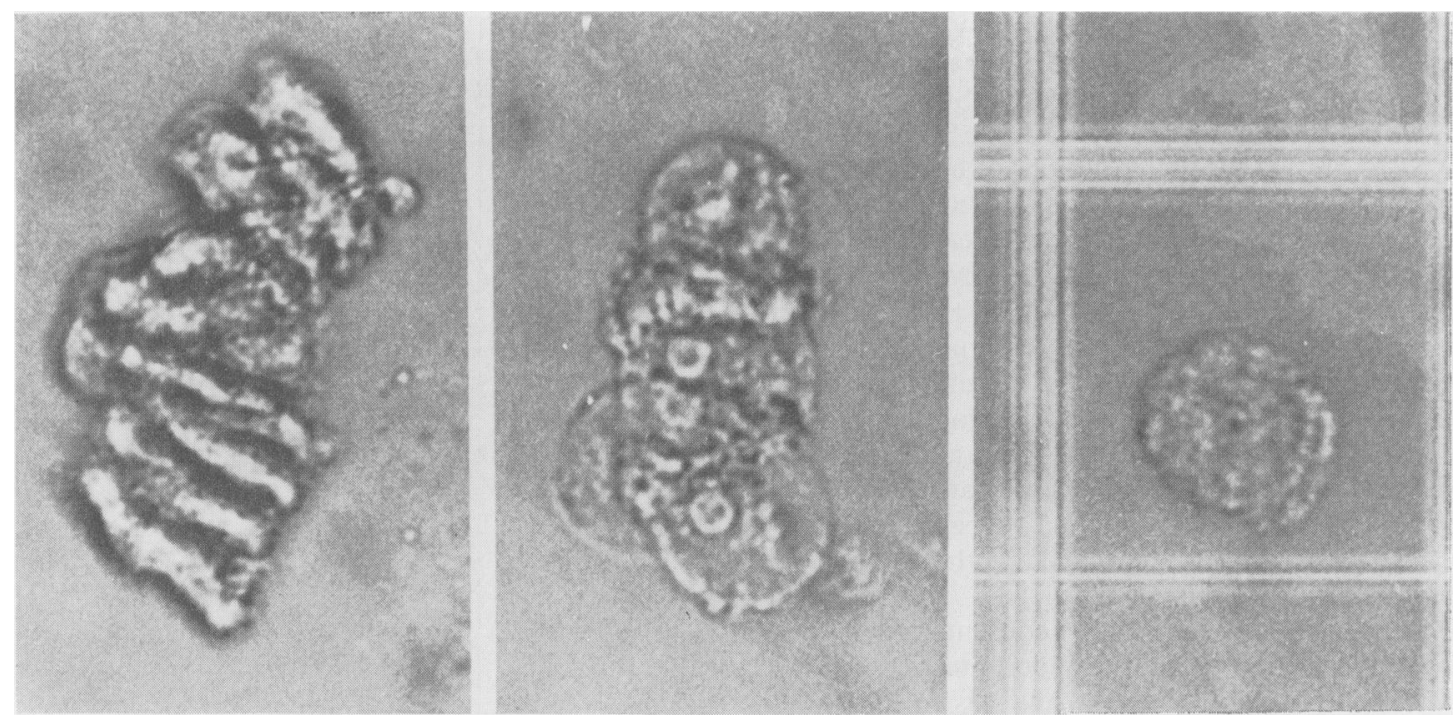

Fig. 1 Colonic epithelial cells, under phase contrast microscopy $(400 \times)$, at different time points during the isolation procedure. (Left) Cells with normal intercellular apposition after one hour of collagenase. (Middle) Cells beginning to lose intercellular junctions after two hours of collagenase. (Right) A typical colonic epithelial cell at the end of the isolation procedure. The short brush border can be seen on the cell.

were washed thoroughly and kept in suspension with complete medium for two hours. Chromium labelling was performed by incubating $1 \times 10^{6}$ cells with $100 \mu \mathrm{Ci}$ ${ }^{51} \mathrm{Cr}$ for 45 minutes at $37^{\circ} \mathrm{C}$. After washing three times in $15 \mathrm{ml}$ of medium, $1 \times 10^{5}$ cells in $150 \mu \mathrm{l}$ complete medium were placed in Eppendorf tubes. One $\times 10^{6}$ PBMC in $150 \mu$ l complete medium were then added and the reaction incubated overnight (10-12 hours) at $37^{\circ} \mathrm{C}$. Control tubes containing only target cells in $300 \mu \mathrm{l}$ of complete medium were used to determine spontaneous release. After incubation the tubes were centrifuged at $900 \mathrm{~g}$ for 10 minutes and the supernatants counted for released ${ }^{51} \mathrm{Cr}$ by gamma spectrometry. Spontaneous release after 12 hours was approximately $30 \%$. Maximum release was obtained by multiple freeze-thaw procedures. SCMC was defined as follows:

test cpm-spontaneous cpm

max. releasable $\mathrm{cpm}-$ spontaneous $\mathrm{cpm}$

PREINCUBATION OF NORMAL PBMC WITH

HEAT INACTIVATED PLASMA

Plasma was obtained from six healthy volunteers and 28 IBD patients. Fourteen had ulcerative colitis and 14 had Crohn's disease. Five of the patients with Crohn's disease had disease limited to the small bowel. Ten patients had severe, nine moderate, and nine mild IBD. Thirteen of the patients from whom plasma was obtained were receiving corticosteroids. The plasma from each subject was divided into multiple $1 \mathrm{ml}$ aliquots and stored at $-70^{\circ} \mathrm{C}$ until use.

Aliquots of frozen plasma were defrosted just before preincubation studies and complement was inactivated by heating for 30 minutes at $56^{\circ} \mathrm{C}$. Heat inactivated plasma $(300 \mu \mathrm{l})$ was added to $4 \times 10^{6}$ normal PBMC suspended in $2 \mathrm{ml}$ DMEM with penicillin and streptomycin and incubated at $37^{\circ} \mathrm{C}$ in a $\mathrm{CO}_{2}$ incubator for either one, 24, or 96 hours. The PBMC were then washed three times in $15 \mathrm{ml}$ of complete medium, recounted and the final concentration adjusted to yield $1 \times 10^{6} \quad \mathrm{PBMC} / 150 \mu \mathrm{l}$ medium. Cytotoxicity against the colon epithelial target cells was assayed as described above. Heat inactivated plasmas without PBMC were also tested in the cytotoxicity assay to determine if plasma carryover might account for colon cell toxicity.

\section{CIRCULATING IMMUNE COMPLEX}

(CIC) LEVELS

Plasma from IBD and control patients were tested for $\mathrm{ClC}$ by Raji cell radioimmune assay as previously described. ${ }^{15}$ Briefly, $25 \mu \mathrm{l}$ of a $1: 4$ dilution of the test plasma were incubated with $3 \times 10^{6}$ Raji cells at $37^{\circ}$ for 45 minutes, followed by washing three times with Hank's buffered salt solution without calcium or magnesium. The washed cells were incubated with $25 \mu \mathrm{l}$ of ${ }^{125} \mathrm{I}$-labelled rabbit antihuman IgG (containing $15 \mu \mathrm{g}$ rabbit IgG), at $4^{\circ} \mathrm{C}$ for 30 minutes. The cells were washed and the amounts of ${ }^{125} \mathrm{I}$ bound to the Raji cells was determined by crystal scintillation 
spectroscopy. The standard curve was constructed with serial dilutions of a pool of sera from patients with systemic lupus erythematosus (SLE) previously shown to contain high levels of CIC. Serial dilutions of SLE sera, rather than dilutions of heat-aggregated human gamma globulin, were used because the former yielded standard curves of greater inter-assay reproducibility and precision. The levels of circulating immune complex present in test samples were expressed as SLE units per $\mathrm{ml}$ of serum. 'SLE units' were established by arbitrarily assigning a value of 10 SLE units per millilitre of serum to the mean CIC level of 42 healthy controls. Values greater than 18 SLE units per $\mathrm{ml}$, two standard deviations above the normal mean, were considered to be abnormal.

The protocol was approved by the Human Experimentation Committee of the Massachusetts General Hospital. Informed consent was obtained from all patients and healthy volunteers.

Wilcoxon nonparametric analysis was used to compare cytotoxicity, direct and induced, in the healthy and IBD patient groups. Associations between CIC levels, steroid therapy, and induction of cytotoxicity were evaluated by $\%^{2}$ analysis.

\section{Results}

PERIPHERAL BLOOD MONONUCLEAR CELL (PBMC) CYTOTOXICITY FOR COLON EPITHELIUM

The cytotoxicity of peripheral blood mononuclear cells from each of the 20 inflammatory bowel disease patients and 16 healthy controls are presented in Fig. 2. PBMC from the IBD group were significantly more toxic for the colonic epithelial cells than were PBMC from the control group $(\mathrm{P}<0.01)$. As can be seen from Fig. 2, however, many IBD patients lacked demonstrable cytotoxicity. Subdivision of the IBD patients according to the method of treatment demonstrated that PBMC from patients not receiving corticosteroid therapy were significantly more cytotoxic for colon epithelium than PBMC from normal controls $(P-0.001)$. In contrast, PBMC from IBD patients receiving corticosteroids did not produce increased cytotoxicity (Fig. 2). Sulphasalazine treatment did not appear to produce a similar suppression of cytotoxicity.

Increased PBMC toxicity for colon epithelium was observed both in patients with ulcerative and granulomatous colonic disease. A patient with granulomatous disease limited to the terminal ileum (by radiographic and sigmoidoscopic examinations) also showed increased cytotoxicity for colon cells.

The increased toxicity of PBMC for colon epithelial cells occurred in patients with mild, moderate, as well as, severe IBD. There was no apparent

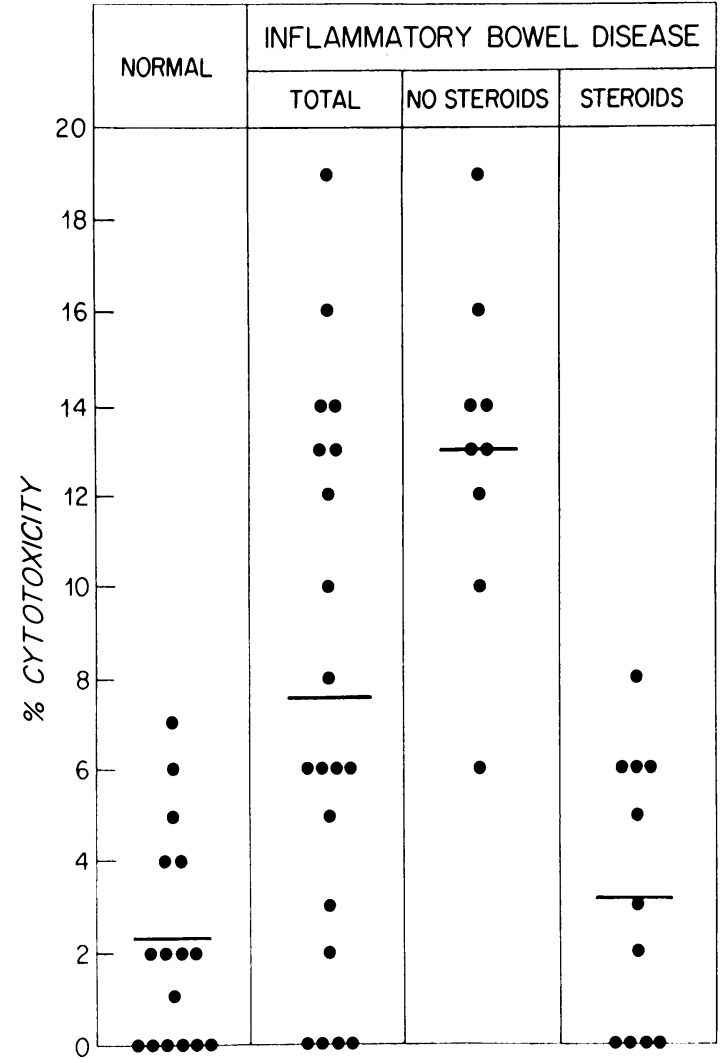

Fig. 2 Peripheral blood mononuclear (ell (PBMC) toxicity for colonic epithelial cells. Each point represents the mean cytotoxicity from triplicate assays performed with PBMC from a normal control or IBD patient. The horizontal lines represent the mean values for the groups. PBMC from IBD patients produced significantly greater cytotoxicity than PBMC from normal controls $(\mathrm{P}<0 \cdot 01)$. Subdivision of IBD patients demonstrates that PBMC from patients not receiving steroids are significantly more cytotoxic than normal PBMC $(\mathrm{P}<0.001)$, while PBMC from patients receiving steroids are not more cytotoxic than normal subjects.

association between disease severity and level of cytotoxicity.

SPONTANEOUS CELL MEDIATED

CYTOTOXICITY (SCMC)

The cytotoxicity demonstrated against colonic epithelial cells by PBMC from untreated IBD patients might have been a reflection of increased nonspecific spontaneous cell mediated cytotoxicity (SCMC). Therefore, we tested PBMC from six healthy controls and nine IBD patients without steroid therapy for their ability to lyse Chang liver cells in vitro. As Table 1 shows, IBD PBMC, at 
Table 1 Spontaneous cell mediated cytotoxicity (SCMC) for Chang liver cells

\begin{tabular}{llll}
\hline Source of PBMC & Subjects (no.) & \multicolumn{2}{l}{ SCMC } \\
\cline { 2 - 3 } & & Mean (\%) & Range (\%) \\
& & $11 \cdot 5$ & $5-19$ \\
Healthy controls & 6 & 12.0 & $4-22$ \\
IBD patients & 9 & & \\
\hline
\end{tabular}

effector:target ratios identical with those used in the colon cytotoxicity studies, displayed normal SCMC levels.

\section{INDUCTION OF PBMC CYTOTOXICITY FOR} COLON EPITHELIUM BY PREINCUBATION WITH HEAT INACTIVATED IBD PLASMA

The results of the induction studies are presented in Fig. 3. Normal PBMC preincubated for 96 hours with inactivated IBD plasma produced significantly greater colon cell toxicity $(P<0.025)$ than PBMC similarly preincubated with normal allogeneic plasma.

To examine the kinetics of this induction, shorter periods of preincubation of normal PBMC with

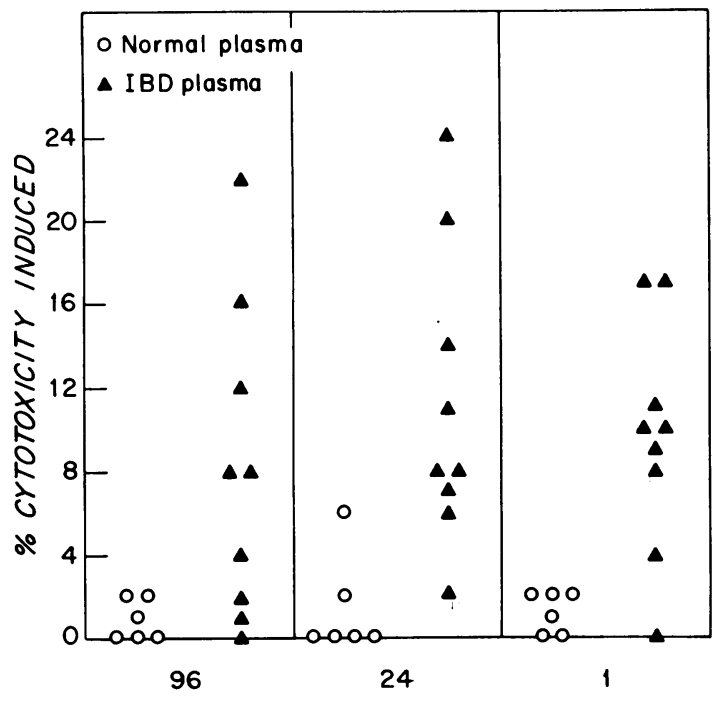

HOURS OF PREINCUBATION WITH PLASMA

Fig. 3 Induction of normal peripheral blood mononuclear cell (PBMC) toxicity for colonic epithelial cells by preincubation with heat inactivated plasma. Each point represents the mean cytotoxicity from triplicate assays performed with normal PBMC after preincubation with six healthy control and nine IBD plasmas for 96, 24 , and one hour periods. The IBD plasmas induced significantly more cytotoxicity at each preincubation period than plasmas from controls $(\mathrm{P}<0.025,<0.005$, and $<0.005$ at 96,24 , and one hours, respectively). heat inactivated plasma were performed. These studies demonstrated that PBMC preincubated with IBD plasma for 24 hours also produced significantly greater colon epithelial cell toxicity $(\mathrm{P}<0.005)$ than the same PBMC preincubated with normal plasma (Fig. 3). In fact, as illustrated in the same figure, IBD plasma pretreatment of normal PBMC for as little as one hour resulted in a statistically significant increase in cytotoxicity $(\mathrm{P}<0.005)$. It should be noted, however, that heat inactivated IBD plasma, in the absence of PBMC, did not produce colon cell toxicity (Table 2).

Unlike the direct cytotoxicity of inflammatory bowel disease PBMC for colon epithelium, the ability of IBD plasma to induce toxicity was not associated with corticosteroid administration. Furthermore, there was no readily apparent association between disease severity and the ability to induce toxicity.

Four of the IBD plasmas which resulted in PBMC cytotoxic activity were fractionated by Sephadex G-200 column chromatography. The capacity to produce PBMC toxicity for colon epithelial cells eluted in the high molecular weight void volume fractions.

Plasma from 18 IBD patients was examined for both its ability to induce PBMC mediated colon cell toxicity and the presence of CIC by Raji RIA. Only three of 10 plasmas inducing cytotoxicity contained detectable CIC. Furthermore, two plasmas with raised CIC lacked induction capability. Thus, there was no association between the induction of cytotoxicity and the presence of raised levels of Raji detectable immune complexes.

\section{Discussion}

The aetiology and pathogenesis of inflammatory bowel disease (IBD) remain unknown. It has been hypothesised that the host immune system is activated, either by external stimuli or by loss of internal suppression, and that it secondarily attacks normal intestinal epithelium. To date the strongest evidence suggesting a role for the immune system in the pathogenesis of IBD has been the demonstration of IBD peripheral blood lymphocyte (PBL) cytotoxicity for trypsinised colon cells in vitro. ${ }^{1-3}$

In the current study, peripheral blood mono-

Table 2 Plasma cytotoxicity for colon epithelial cells

\begin{tabular}{lcll}
\hline Source of plasma & Subjects (no.) & \multicolumn{2}{l}{ Plasma cytotoxicity } \\
\cline { 2 - 4 } & & Mean (\%) & Range (\%) \\
\hline Healthy controls & 7 & $0 \cdot 3$ & $0-2$ \\
IBD patients & 12 & $0 \cdot 8$ & $0-6$ \\
\hline
\end{tabular}


nuclear cells (PBMC) from patients with IBD again produced significantly greater in vitro toxicity for colon epithelial cells than did PBMC from healthy controls. The colon epithelial cells in this study were obtained by mechanical separation and gentle collagenase digestion. Thus, these results not only confirm published findings, but also suggest that the reported cytotoxicity is unrelated to trypsin induced changes in the colon cell surface membrane.

Although the PBMC cytotoxicity for colon cells was significantly increased in the IBD patient group, PBMC from some IBD patients showed no demonstrable cytotoxicity. Patients failing to display cytotoxicity were primarily those receiving corticosteroid therapy, while patients with increased colon cell toxicity were receiving either other forms of therapy or no treatment for their IBD. These data suggest that steroids administered in vivo may block IBD PBMC toxicity for colon epithelium in vitro. Although the mechanism of this effect was not clearly defined, the above results, coupled with the apparent lack of effect of steroids on induction capabilities of IBD plasma, suggest several potential mechanisms. Steroids may decrease the absolute number of cells capable of undergoing activation, inhibit the ability of lymphocytes to be armed in vivo, or suppress the cytotoxic expression of activated lymphocytes. They apparently do not decrease the production of arming factor(s).

The cytotoxic activity of PBMC did not correlate with the clinical activity of IBD. A similar lack of correlation was reported in a study in which a single individual was followed over a seven month period of variable clinical activity. ${ }^{16}$ The reported loss of cytotoxicity 10 days after resection of diseased bowel $^{34}$ initially appears to be inconsistent with the above findings. However, while those results suggest that diseased bowel must be present for cytotoxicity to be demonstrable, they do not really provide direct evidence regarding the relationship of severity of clinical disease and toxicity of IBD lymphocytes for colon epithelial cells.

It is unclear precisely how the in vitro assays relate to the pathogenesis of IBD. In our study, and in the studies of Shorter et al., ${ }^{34}$ patients with Crohn's disease as well as those with ulcerative colitis had PBMC toxicity for colon epithelial cells in vitro. In fact, PBMC from one of our patients with Crohn's disease limited to the small bowel displayed one of the highest levels of colon cell toxicity. One possible explanation is that the patient with regional enteritis has subclinical disease distal to the small bowel. As clinical methods for detection of disease are relatively crude, a substantial number of patients with no radiographic or sigmoidoscopic evidence of large bowel disease may indeed have mild sub- clinical colonic involvement. Alternatively, it is possible that the same cytotoxic potential actually occurs in both ulcerative colitis and Crohn's disease but that local cellular or humoral factors alter or regulate the immunological expression in vivo.

The restriction of in vitro colon cell toxicity to lymphocytes obtained from patients with $\mathrm{IBD}^{17}$ has been important in implicating cellular immune responses in the pathogenesis of IBD. However, equally important is the demonstration that such toxicity does not merely reflect a generalised increase in spontaneous cell toxicity for cultured human cells. The lack of toxicity of IBD PBMC for Chang liver cells documented in this study suggests that the cytotoxicity for colon cells is not due to increased SCMC in IBD patients.

The present results indicate that heat inactivated plasma from some IBD patients, though itself not toxic, is capable of inducing normal PBMC to become toxic for colon epithelial cells in culture, after 96 hours of preincubation. Furthermore, some IBD plasmas can also induce toxicity after considerably shorter preincubation periods. These rapid induction results are in contrast with the reports of Perlmann and Broberger ${ }^{1}$ and Shorter et al. ${ }^{4}$ We used plasma rather than serum for induction, but this difference is not likely to be responsible for the disparate results. The most likely explanation for the disparity may be the differences in the cytotoxicity assays. The target cells are prepared differently and thus the population of cells, their antigenic determinants and their susceptibility to lysis may be dissimilar. Furthermore, the effector:target cell ratio, a parameter known to be of great importance in in vitro cytotoxicity studies, ${ }^{18}$ was greater in our experiments than either of the other two investigations. If only a small percentage of the total effector cell population were inducible, the other studies may have had less than optimal 'induced effector': target cell ratios for observing cytotoxicity. Finally, our assay incubation was performed overnight compared with four hours or less in the other studies. As it is known that certain effector cells can make multiple 'hits', ${ }^{10} 20$ the increased reaction time may have allowed an amplification phenomenon to occur in our system to produce identifiable cytotoxicity by very few 'induced effector' cells. Studies performed over shorter periods would lack such an amplification and therefore fail to demonstrate toxicity.

The previously demonstrated loss of cytotoxicity after colectomy ${ }^{34}$ is most compatible with CIC activation and direction of normal PBL, as complexes, but not specific antibody or committed $\mathrm{T}$ cells, would be expected to disappear rapidly after resection. The high molecular weight of the in- 
ducing substance', as suggested by gel chromatography, is also consistent with immune complex induction of cytotoxicity. To further examine the role of CIC, plasmas examined for the induction of cytotoxicity were also examined for the presence of CIC by a Raji cell radioimmunoassay. This technique, which measures a wide range of complement fixing CIC with considerable sensitivity, ${ }^{21}$ demonstrated complexes in several patients. However, there was no demonstrable association between the presence of CIC and the induction capabilities of plasma samples.

Complexes which do not activate the complement sequence or are relatively small in size will not be detected by the Raji radioimmunoassay. ${ }^{21}$ Therefore, while the above data exclude a large population of complexes, they do not preclude the possibility that a particular subset of CIC might be responsible for the induction of cytotoxicity. Further characterisation of the inducing factor should lead to a better understanding of its possible role in the immunopathogenesis of IBD.

The authors would like to acknowledge the excellent technical assistance of Kathy O'Loughlin and Nancy Neyhard and to thank the surgical and pathology staffs of the Massachusetts General Hospital whose cooperation made the study possible. This work was supported in part by grants from the National Foundation for Ileitis and Colitis and the National Institutes of Health (AM07191). This work was presented in part at the 7 May 1979 meeting of the American Society for Clinical Investigation.

\section{References}

${ }^{1}$ Perlmann $\mathrm{P}$, Broberger $\mathrm{O}$. In vitro studies of ulcerative colitis. II. Cytotoxic action of white blood cells from patients on human fetal colon cells. J Exp Med 1963; 117: $717-33$

${ }^{2}$ Watson DW, Quigley A, Bolt RJ. Effect of lymphocytes from patients with ulcerative colitis on human adult colon epithelial cells. Gastroenterology 1966; 51 : 985-93. 'Shorter RG, Cardoza M, Spencer RJ, Huizenga KA. Further studies of in vitro cytotoxicity of lymphocytes from patients with ulcerative and granulomatous colitis for allogeneic colonic epithelial cells, including the effects of colectomy. Gastroenterology 1969; 56: 304-9.

'Shorter RG, Huizenga KA, ReMine SG, Spencer RJ. Effects of preliminary incubation of lymphocytes with serum on their cytotoxicity for colonic epithelial cells. Gastroenterology 1970; 58: 843-50.

"Shorter RG, Huizenga KA, Spencer RJ, Aas J, Guy SK. Inflammatory bowel disease. Cytophilic antibody and the cytotoxicity of lymphocytes for colonic cells in vitro Am J Dig Dis 1971; 16: 673-80.
${ }^{6}$ Perlmann P, Perlmann H, Biberfeld P. Specifically cytotoxic lymphocytes produced by preincubation with antibody-complexed target cells. J Immunol 1972; 108: 558-61.

${ }^{7}$ Parrilllo JE, Fauci AS. Apparent direct cellular cytotoxicity mediated via cytophilic antibody. Multiple Fc receptor bearing effector cell populations mediating cytophilic antibody induced cytotoxicity. Immunology 1977; 33: 839-50.

${ }^{8}$ Stobo JD, Tomasi TB, Huizenga KA, Spencer RJ, Shorter RG. In vitro studies of inflammatory bowel disease. Surface receptors of the mononuclear cell required to lyse allogeneic colonic epithelial cells. Gastroenterology 1976; 70: 171-6.

9 Jewell DP, MacLennan ICM. Circulating immune complexes in inflammatory bowel disease. Clin Exp Immunol 1973; 14: 219-26.

${ }^{10}$ Hodgson HJF, Potter BJ, Jewell DP. Immune complexes in ulcerative colitis and Crohn's disease. Clin Exp Immunol 1977; 29: 187-96.

${ }^{11}$ Kemler BJ, Alpert E. Inflammatory bowel disease associated circulating immune complexes. Gut (In press).

${ }^{12}$ Truelove SC, Witts LJ. Cortisone in ulcerative colitis: final report on a therapeutic trial. $B r$ Med $J$ 1955; 2: 1041-8.

${ }^{13}$ deDombal FT, Burton IL, Clamp SE, Goligher JC. Short-term course and prognosis in Crohn's disease. Gut 1974; 15: 435-43.

${ }^{14}$ Boyum A. Isolation of mononuclear cells and granulocytes from human blood. Scand J Clin Lab Invest 1968; 21: suppl 97, 77-89.

${ }^{15}$ Kemler BJ, Franklin WD, Alpert E, Bloch KJ. Failure to detect circulating immune complexes in allergic patients on injection therapy. Clin Allergy 9: 473-8.

${ }^{16}$ Watson DW, Quigley A, Bolt RJ. The cytotoxicity of circulating lymphocytes from ulcerative colitis patients for human colon epithelial cells: Disease specificity and relationship to disease activity. (Abstract.) Gastroenterology 1966; 50: 886-7.

${ }^{17}$ Shorter RG, Tomasi TB, Huizenga KA, Spencer RJ, Stobo JD. The immunology of chronic ulcerative colitis and Crohn's disease. Ann NY Acad Sci 1976; 278: 586-91.

${ }^{18}$ Holm G, Perlmann P. Quantitative studies on phytohaemagglutinin-induced cytotoxicity by human lymphocytes against homologous cells in tissue culture. Immunology 1967; 12: 525-36.

${ }^{19}$ Zagury D, Bernard J, Thierness N, Feldman M, Berke $G$. Isolation and characterization of individual functionally reactive cytotoxic $\mathrm{T}$ lymphocytes: conjugation, killing and recycling at the single cell level. Eur $J$ Immunol 1975; 5: 818-22.

${ }^{20}$ Martz E. Multiple target cell killing by the cytolytic T lymphocyte and the mechanism of cytotoxicity. Transplantation 1976; 21 : 5-11.

${ }^{21}$ Lambert PH, Dixon FJ, Zubler RH. A WHO collaborative study for the evaluation of eighteen methods for detecting immune complexes in serum. J Clin Lab Immunol 1978; 1: 1-15. 